\title{
Electromagnetic Green's functions retrieval by cross-correlation and cross-convolution in media with losses
}

\author{
Evert Slob $^{1}$ and Kees Wapenaar ${ }^{1}$ \\ Received 15 December 2006; revised 31 January 2007; accepted 7 February 2007; published 13 March 2007.
}

[1] It is shown that the electromagnetic Green's functions of any linear medium with arbitrary heterogeneity can be obtained from the cross-correlation, or the crossconvolution, of two recordings at different receiver locations in an open system. Existing representations are known for cross-correlations where time-reversal invariance is exploited and hence they are considered in lossless media. We show here that the cross-correlation type representations are exact in a configuration with sources on a closed boundary and the medium has non-zero loss terms only outside this boundary. Furthermore, we show that for crossconvolution representations the loss mechanisms may exist anywhere in space. Many sources of electromagnetic signals exist in the atmosphere and in populated areas, and these can be used in a large variety of practical passive applications exploiting eddy current or electromagnetic wave techniques. Citation: Slob, E., and K. Wapenaar (2007), Electromagnetic Green's functions retrieval by cross-correlation and cross-convolution in media with losses, Geophys. Res. Lett., 34, L05307, doi:10.1029/2006GL029097.

\section{Introduction}

[2] Retrieving the Green's function between two points from correlating recorded transients or noise fields has been formulated theoretically [Claerbout, 1968; Cox, 1973; Weaver and Lobkis, 2001, 2004; Wapenaar, 2003, 2004; van Tiggelen, 2003; Malcolm et al., 2004; Snieder, 2004; Roux et al., 2005; Wapenaar and Fokkema, 2006] and demonstrated experimentally [Lobkis and Weaver, 2001; Derode et al., 2003; Roux et al., 2004; Larose et al., 2006; Draganov et al., 2006a] using open, half open and closed systems (e.g. wave guides or irregular finite bodies). In open systems the wave field must be diffuse in random media or be generated by a continuum of transient or noise sources on a closed surface in deterministic media. In half open and closed media we can rely on the mirror function of the closed sides. The idea has been applied to microearthquake data [Daneshvar et al., 1995], obtaining the shear wave velocity structure in the first $100 \mathrm{~m}$ of the Earth [Louie, 2001], imaging the interior structure of the Sun [Ricket and Claerbout, 1999], imaging the internal structure of the Earth's crust [Campillo and Paul, 2003; Shapiro and Campillo, 2004; Sabra et al., 2005; Shapiro et al., 2005], imaging hydrocarbon reservoirs [Schuster et al., 2004], for detecting under water objects [Buckingham et al., 1992; Epifanio et al., 1999] and a weighted version in structural mechanics [Snieder et al.,

\footnotetext{
${ }^{1}$ Department of Geotechnology, Delft University of Technology, Delft, Netherlands.
}

Copyright 2007 by the American Geophysical Union. 0094-8276/07/2006GL029097\$05.00 2006a]. Time-reversal invariance is used by all and hence most theories apply only to lossless wave propagation, except for Roux et al. [2005] who treated a single homogeneous lossy space theoretically. A general, cross-correlation based, formulation for lossy media involving volume sources as proposed by Snieder [2006] is presented by Wapenaar et al. [2006].

[3] We use the same reaction principles as Bojarski [1983] and explicitly address the issue of loss factors, because many materials have non-zero electric conductivity values and/or show polarization or other dispersion. In addition to the reciprocity theorem of the time-correlation type we use the time-convolution type to derive a Green's function representation. Slob et al. [2006a] studied the effect of neglecting the loss factors in the formulation. Here we derive the general formulation including all possible loss factors and give explicit examples how the Green's function of a lossy medium can be retrieved exactly with sources distributed on a surface and show how approximations to the exact results leads to practical applications. The reciprocity theorem is applied to Maxwell's equations for two non-identical states that could exist in the same medium and occupy the same domain. This leads to a general Green's function representation for electromagnetic fields and waves between two points. The two points can be inside this source boundary, or one point can be outside. In the first situation, the representation for the causal electric field Green's function is obtained together with its time-reversed counterpart and is equivalent to known acoustic and elastodynamic representations [Wapenaar and Fokkema, 2006]. In the second situation, representations for the causal Green's function are obtained. In addition we show here how the electromagnetic reciprocity theorem of the timeconvolution type leads to the causal Green's function without constraints on the loss mechanisms. Examples of possible sources are various human activities (e.g. VLF radio and wireless communication systems) and background radiation, while passive applications can be found in ground penetrating radar applications, material characterization, eddy current techniques and super resolution focusing.

\section{Green's Function Representations}

[4] We use the subscript notation to denote Cartesian vectors and tensors. All used subscripts denote vector components in the Cartesian reference frame. The spacetime electromagnetic field is determined by the electric field vector components $E_{k}(\boldsymbol{x}, t)$ in the $x_{k}$-direction, $k=1,2,3$, the magnetic field $H_{j}(\boldsymbol{x}, t)$, and the external source volume densities of electric and magnetic currents, $\left\{J_{k}^{e}(\boldsymbol{x}, t), J_{j}^{m}(\boldsymbol{x}\right.$, $t)\}$, respectively. The medium parameters of any heterogeneous and anisotropic medium can be represented by the 
rank-two tensors for the electric permittivity and conductivity, $\left\{\varepsilon_{k r}(\boldsymbol{x}), \sigma_{k r}^{e}(\boldsymbol{x}, t)\right\}$, and for the magnetic permeability and conductivity, $\left\{\mu_{j p}(\boldsymbol{x}), \sigma_{j p}^{m}(\boldsymbol{x}, t)\right\}$, where the conductivities are time-convolution operators. The permittivity and permeability are defined as functions of position only. This is no restriction because the time dependence of these medium parameters can be incorporated in the conductivities. The time-Fourier transform of a space-time dependent quantity is defined as $\hat{f}(\boldsymbol{x}, \omega)=\int \exp (-\mathrm{j} \omega t) f(\boldsymbol{x}, t) \mathrm{d} t$, where $\mathrm{j}$ is the imaginary unit and $\omega$ denotes angular frequency. In the space-frequency domain Maxwell's equations in matter are given by $-\epsilon_{k m j} \partial_{m} \hat{H}_{j}+\left[\hat{\sigma}_{k r}^{e}+\mathrm{j} \omega \varepsilon_{k r}\right] \hat{E}_{r}=$ $-\hat{J}_{k}^{e}$, and $\epsilon_{j m r} \partial_{m} \hat{E}_{r}+\left[\hat{\sigma}_{j p}^{m}+\mathrm{j} \omega \mu_{j p}\right] \hat{H}_{p}=-\hat{J}_{j}^{m}$, where $\partial_{m}$ denotes partial differentiation with respect to the coordinate $x_{m}$ and $\epsilon_{k m j}$ is the anti-symmetric tensor of rank three, $\epsilon_{k m j}=1$ when $k m j=\{123,231,312\}, \epsilon_{k m j}=-1$ when $k m j=\{132$, $213,321\}$, while $\epsilon_{k m j}=0$ otherwise. The corresponding time-correlation interaction quantity is given by $\epsilon_{m k j}\left(\hat{E}_{k, A}^{*} \hat{H}_{j, B}+\hat{E}_{k, B} \hat{H}_{j, A}^{*}\right)$, where the asterisk denotes complex conjugation and state $\mathrm{A}$ is the time-reversed causal state, while state B is a, different, causal state. For reciprocal media all material property tensors are symmetric and we take equal material parameters for the states $\mathrm{A}$ and $\mathrm{B}$. We take the divergence of the interaction quantity and substitute the result in Maxwell's equations. Then we integrate over a bounded domain $\mathbb{D}$, with closed boundary $\partial \mathbb{D}$ and outward pointing unit normal vector $n_{j}$ and apply Gauss' divergence theorem to obtain

$$
\begin{aligned}
& \oint_{\boldsymbol{x} \in \partial \mathbb{D}} n_{m} \epsilon_{m k j}\left(\hat{E}_{k, A}^{*} \hat{H}_{j, B}+\hat{E}_{k, B} \hat{H}_{j, A}^{*}\right) \mathrm{d}^{2} \boldsymbol{x} \\
& =-2 \int_{\boldsymbol{x} \in \mathbb{D}}\left[\hat{H}_{j, A}^{*} \Re\left\{\hat{\sigma}_{j p}^{m}\right\} \hat{H}_{p, B}+\hat{E}_{k, A}^{*} \Re\left\{\hat{\sigma}_{k r}^{e}\right\} \hat{E}_{r, B}\right] \mathrm{d}^{3} \boldsymbol{x} \\
& \quad-\int_{\boldsymbol{x} \in \mathbb{D}}\left[\left(\hat{J}_{r, A}^{e}\right)^{*} \hat{E}_{r, B}+\hat{J}_{k, B}^{e} \hat{E}_{k, A}^{*}+\hat{J}_{j, B}^{m} \hat{H}_{j, A}^{*}+\left(\hat{J}_{p, A}^{m}\right)^{*} \hat{H}_{p, B}\right] \mathrm{d}^{3} \boldsymbol{x} .
\end{aligned}
$$

The domain $\mathbb{D}$ with boundary $\partial \mathbb{D}$ is a subdomain of the open configuration; the boundary does in general not coincide with a physical boundary. The complement of $\mathbb{D}$ and $\partial \mathbb{D}$ is denoted $\mathbb{D}^{\prime}$.

[5] The corresponding time-convolution interaction quantity is given by $\epsilon_{m k j}\left(\hat{E}_{k, A} \hat{H}_{j, B}-\hat{E}_{k, B} \hat{H}_{j, A}\right)$. Repeating the same procedure gives

$$
\begin{aligned}
& \oint_{\boldsymbol{x} \in \partial \mathbb{D}} n_{m} \epsilon_{m k j}\left(\hat{E}_{k, A} \hat{H}_{j, B}-\hat{E}_{k, B} \hat{H}_{j, A}\right) \mathrm{d}^{2} \boldsymbol{x} \\
& =\int_{\boldsymbol{x} \in \mathbb{D}}\left[\left(\hat{J}_{r, A}^{e}\right) \hat{E}_{r, B}-\hat{J}_{k, B}^{e} \hat{E}_{k, A}+\hat{J}_{j, B}^{m} \hat{H}_{j, A}-\left(\hat{J}_{p, A}^{m}\right) \hat{H}_{p, B}\right] \mathrm{d}^{3} \boldsymbol{x} .
\end{aligned}
$$

For a more detailed discussion on reciprocity relations, see de Hoop [1995]. What is interesting to note here is that the material properties that contribute to the wave energy loss, the conductivities $\sigma^{e, m}$, are present in equation (), while they are not in equation (2).

[6] Next we specify source positions by taking non-zero electric source currents as point sources, $\hat{J}_{k}^{e},\{A, B\}(\boldsymbol{x}, \omega)=$ $\delta_{k r} \delta\left(\boldsymbol{x}-\boldsymbol{x}_{\{A, B\}}\right), \delta\left(\boldsymbol{x}-\boldsymbol{x}_{\{A, B\}}\right)$ being the 3D Dirac function and $\delta_{k r}$ is the Kronecker delta function. Then the electric and magnetic fields reduce to Green's functions
$\hat{E}_{k,\{A, B\}}(\boldsymbol{x}, \omega)=\hat{G}_{k r,\{A, B\}}^{E e}=\hat{G}_{k r}^{E e}\left(\boldsymbol{x}, \boldsymbol{x}_{\{A, B\}}, \omega\right)=\hat{G}_{r k}^{E e}\left(\boldsymbol{x}_{\{A, B\}}\right.$, $\boldsymbol{x}, \omega), \hat{H}_{j,\{A, B\}}(\boldsymbol{x}, \omega)=\hat{G}_{j r,\{A, B\}}^{H e}=\hat{G}_{j r}^{H e}\left(\boldsymbol{x}, \boldsymbol{x}_{\{A, B\}}, \omega\right)=$ $-\hat{G}_{r j}^{E m}\left(\boldsymbol{x}_{\{A, B\}}, \boldsymbol{x}, \omega\right)$, where the first superscript denotes the field and the second denotes the source type, $\hat{G}_{r j}^{E m}$ denotes the electric field Green's function due to a magnetic current source and source-receiver reciprocity has been used. The points $\boldsymbol{x}, \boldsymbol{x}_{A}, \boldsymbol{x}_{B}$ can be anywhere in space. Substituting all our choices in equation (1) results in

$$
P_{A B}^{(r)}=P_{A B}^{(i n)}+\dot{W}_{A B}^{(h)},
$$

where

$$
P_{A B}^{(r)}=\hat{G}_{k r, A B}^{E e} \chi_{\mathbb{D}}\left(x_{A}\right)+\left\{\hat{G}_{k r, A B}^{E e}\right\}^{*} \chi_{\mathbb{D}}\left(\boldsymbol{x}_{B}\right),
$$

$$
P_{A B}^{(i n)}=\oint_{\boldsymbol{x} \in \partial \mathbb{D}}\left(\epsilon_{m p j} n_{m}\left\{\hat{G}_{k p, A}^{E e}\right\} *\left\{\hat{G}_{r j ; B}^{E m}\right\}+\epsilon_{m p j} n_{m}\left\{\hat{G}_{k j, A}^{E m}\right\} *\left\{\hat{G}_{r p, B}^{E e}\right\}\right) \mathrm{d}^{2} \boldsymbol{x}
$$

$$
\begin{aligned}
\dot{W}_{A B}^{(h)}= & 2 \int_{\boldsymbol{x} \in \mathbb{D}}\left[\left\{\hat{G}_{k j, A}^{E m}\right\}^{*} \Re\left\{\hat{\sigma}_{j p}^{m}\right\} \hat{G}_{r p, B}^{E m}\right. \\
& \left.+\left\{\hat{G}_{k m, A}^{E e}\right\}^{*} \Re\left\{\hat{\sigma}_{m n}^{e}\right\} \hat{G}_{r n, B}^{E e}\right] \mathrm{d}^{3} \boldsymbol{x} .
\end{aligned}
$$

The notation is chosen to resemble Poynting's theorem in the case $\boldsymbol{x}_{B}=\boldsymbol{x}_{A}$. For that situation, $P_{A A}^{(r)}$ denotes the total power received at the point $\boldsymbol{x}_{A}, P_{A A}^{(i n)}$ is the total instantaneous power that is generated at $\partial \mathbb{D}$ and transferred to the point $\boldsymbol{x}_{A}$, while $\dot{W}_{A A}^{(h)}$ represents the total dissipated power from $\partial \mathbb{D}$ to $\boldsymbol{x}_{A}$. For different locations of $\boldsymbol{x}_{A}$ and $\boldsymbol{x}_{B}, \hat{G}_{k r, A B}^{E e}=\hat{G}_{k r}^{E e}\left(\boldsymbol{x}_{A}, \boldsymbol{x}_{B}, \omega\right)$ in the right-hand side of equation (4) denotes the Green's function between the points $\boldsymbol{x}_{A}$ and $\boldsymbol{x}_{B}$, while the characteristic function of the domain $\mathbb{D}$ is defined as $\chi_{\mathbb{D}}(\boldsymbol{x})=\{0,1 / 2,1\}$ for $\left.\boldsymbol{x} \in \mathbb{D}^{\prime}, \partial \mathbb{D}, \mathbb{D}\right\} . P_{A B}^{(r)}$ vanishes when both points $\boldsymbol{x}_{A}$ and $\boldsymbol{x}_{B}$ are located in $\mathbb{D}^{\prime}$. When the point $\boldsymbol{x}_{A}$ is inside $\mathbb{D}$ the causal Green's function is obtained and when $\boldsymbol{x}_{B}$ is inside $\mathbb{D}$ the time-reversed causal Green's function is obtained. The Green's functions in the right-hand side of equations (5) and (6) represent measured electric fields at the points $\boldsymbol{x}_{A}$ and $\boldsymbol{x}_{B}$ generated by electric and magnetic sources located on the surface. Equation (3) implies that we can retrieve the Green's function of any linear response medium, represented by $P^{(r)}$, if electric and magnetic sources exist on the surface, whose contributions then represent $P^{(i n)}$, and when $\dot{W}^{(h)}$ is known. Together, equations (3)-(6) form the exact time-correlation type interferometric representation for the electric field due to an electric current source.

[7] The term $\dot{W}^{(h)}$ vanishes when the electric and magnetic conductivity functions are non-zero only in $\mathbb{D}^{\prime}$. By taking zero conductivities inside $\mathbb{D}$ and both $\boldsymbol{x}_{A}$ and $\boldsymbol{x}_{B}$ inside $\mathbb{D}$, equations (3)-(6) represent the electromagnetic equivalent of the acoustic and elastodynamic representations of Wapenaar and Fokkema [2006]. 


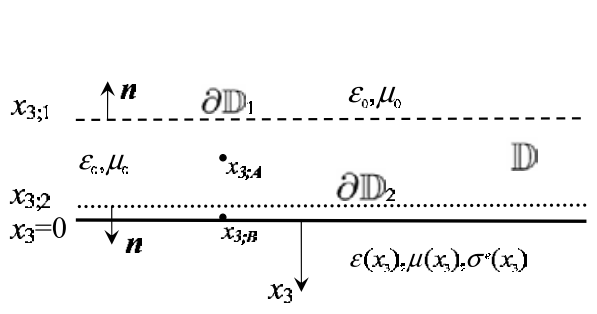

(a)

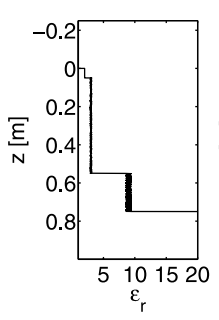

(b)

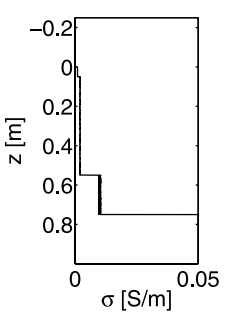

(c)

Figure 1. (a) A $1 \mathrm{D}$ example configuration with sources at $\partial \mathbb{D}_{1}, x_{3}=x_{3 ; 1}$, but no sources at $\partial \mathbb{D}_{2}, x_{3}=x_{3 ; 2}$, with outward unit normal vector, $\boldsymbol{n}$, one receiver at $\boldsymbol{x}_{\mathbf{3} ; A}$ in a homogeneous medium and one at the Earth surface at $\boldsymbol{x}_{\mathbf{3} ; B}$ with a heterogeneous and lossy medium below the surface. The depth profiles of (b) the electric permittivity and (c) the conductivity.

[8] To avoid the presence of a volume integral in the representation, we substitute the same choices in equation (2) to find,

$$
\begin{aligned}
& \hat{G}_{k r, A B}^{E e}\left[\chi_{\mathbb{D}}\left(\boldsymbol{x}_{A}\right)-\chi_{\mathbb{D}}\left(\boldsymbol{x}_{B}\right)\right]= \\
& \quad \oint_{\boldsymbol{x} \in \partial \mathbb{D}} \epsilon_{m p j} n_{m}\left(\hat{G}_{k p, A}^{E e} \hat{G}_{r j, B}^{E m}-\hat{G}_{k j, A}^{E m} \hat{G}_{r p, B}^{E e}\right) \mathrm{d}^{2} \boldsymbol{x}
\end{aligned}
$$

where it is clear from the left-hand side that the exact causal Green's function is obtained only when one point is inside and the other is outside $\mathbb{D}$. Equation (7) is the exact timeconvolution type interferometric representation of the electric field in the $x_{k}$-direction at $\boldsymbol{x}_{A}$ due to an electric current source in the $x_{r}$-direction located at $\boldsymbol{x}_{B}$. The righthand side expresses that the Green's function is obtained from cross-convolving electric fields recorded at $\boldsymbol{x}_{A}$ and $\boldsymbol{x}_{B}$ due to electric and magnetic current sources at the boundary $\partial \mathbb{D}$ and then summed over all sources on the closed surface. Equations (3)-(6) and (7) are exact and show how the electric field due to an electric current source can be obtained from either cross-correlations or crossconvolutions of observed electric fields at two points. By taking current sources of the magnetic current type for both states in equation (1) and (2), similar representations for the exact magnetic field Green's function due to a magnetic current source are obtained. If in state $A$ an electric current source is taken and in state $B$ a magnetic current source, the electric field Green's function due to a magnetic current source is obtained.

\section{Modifications for Practical Applications}

[9] In equations (5)-(7) both electric and magnetic current sources are assumed to be present on the whole surface. This restricts applications using uncontrolled sources because both source types need to be present and we need to measure their effects separately. Our first step toward a more practical representation is to reformulate the right-hand sides in both equations in terms of electric current sources only. This is achieved by rewriting $\hat{G}^{E m}$ in equations (5) and (6) as $\hat{G}_{r j}^{E m}\left(\boldsymbol{x}_{\{A, B\}}, \boldsymbol{x}, \omega\right)=-\hat{G}_{j r}^{H e}\left(\boldsymbol{x}, \boldsymbol{x}_{\{A, B\}}\right.$, $\omega)=(\mathrm{i} \omega \mu(\boldsymbol{x}))^{-1} \epsilon_{j m k} \partial_{m} \hat{G}_{r k}^{E e}\left(\boldsymbol{x}_{\{A, B\}}, \boldsymbol{x}, \omega\right)$. After this step the integrand contains a dipole and quadrupole response [Slob et al., 2006a]. Under the assumption that the medium in the neighborhood of the boundary is smoothly varying and isotropic, the quadrupole electric current sources are normal derivatives of dipole sources. In the far-field approximation $(\mathrm{i} \omega \mu)^{-1} \epsilon_{n p j} n_{n} \epsilon_{j m k} \partial_{m} \hat{G}_{r k}^{E e} \approx-(c \mu)^{-1} \hat{G}_{r p}^{E e}$. Substitution in equation (3) yields

$$
\begin{aligned}
& \hat{G}_{k r, A B}^{E e} \chi_{\mathbb{D}}\left(\boldsymbol{x}_{A}\right)+\left\{\hat{G}_{k r, A B}^{E e}\right\}^{*} \chi_{\mathbb{D}}\left(\boldsymbol{x}_{B}\right)= \\
& -\frac{2}{\mu c} \oint_{\boldsymbol{x} \in \partial \mathbb{D}}\left\{\hat{G}_{k j, A}^{E e}\right\}^{*} \hat{G}_{r j, B}^{E e} \mathrm{~d}^{2} \boldsymbol{x}+{ }^{\prime} \text { ghost }^{\prime} .
\end{aligned}
$$

This representation leads to practical formulations when transient or mutually uncorrelated sources are used [Wapenaar, 2004; Slob et al., 2006b; Wapenaar and Fokkema, 2006]. The ghost-term contains all contributions from waves that initially travel inward from the source boundary and are recorded at $\boldsymbol{x}_{A}$, which interact with initially outward traveling waves that are recorded at $\boldsymbol{x}_{\boldsymbol{B}}$, and whose combined interactions vanish in the exact representation, but which do not lead to complete destructive interference in the far-field approximation. While for a smooth boundary the ghost-term is non-zero, it will vanish for irregular distribution of sources [Draganov et al., 2004, 2006b]. The reason is that the ghost-term consists of events for which source position dependent amplitude errors are made, which can lead to non-physical events in the final result.

[10] Considering a smooth medium near the source surface and far field conditions in the exact time-convolution type representation leads to

$$
\begin{gathered}
\hat{G}_{k r}^{E e}\left(\boldsymbol{x}_{A}, \boldsymbol{x}_{B}, \omega\right)\left|\chi_{\mathbb{D}}\left(\boldsymbol{x}_{A}\right)-\chi_{\mathbb{D}}\left(\boldsymbol{x}_{B}\right)\right|= \\
\frac{2}{\mu c} \oint_{\boldsymbol{x} \in \partial \mathbb{D}} \hat{G}_{k j, A}^{E e} \hat{G}_{r j, B}^{E e} \mathrm{~d}^{2} \boldsymbol{x}+{ }^{\prime} \text { ghost }^{\prime} .
\end{gathered}
$$

This representation leads to practical formulations when transient sources are used.

[11] One of the configurations that can be used in the Earth sciences is to take the domain $\mathbb{D}$ in the air, where we can have two receivers in this domain or one inside and one outside. The Earth is a heterogeneous, anisotropic and lossy medium, for which accurate reflection electric field data can be created by cross-correlating recorded electric field components from transient or mutually uncorrelated noise sources at the two surfaces. In this configuration, the sources at the boundary furthest away from the Earth surface (upper boundary) gives all the physical contributions, while the other boundary gives 'ghost events' and 


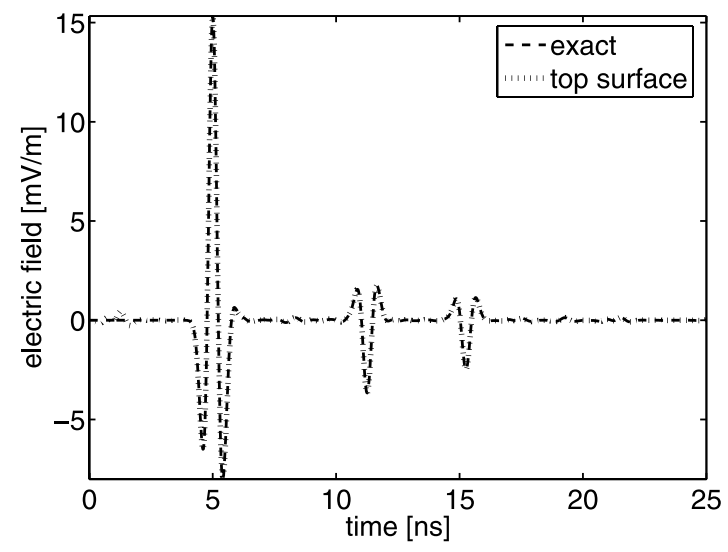

(a)

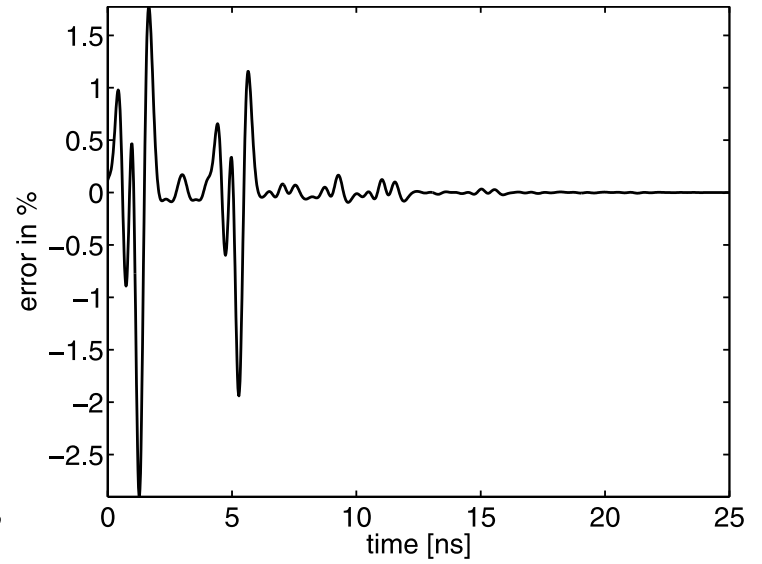

(b)

Figure 2. Cross-correlation result from the configuration in Figure 1 plot together with the exact result (a), and (b) the error between the two curves in \% normalized to the maximum amplitude of the exact response.

corrects for the amplitudes of physical events that are not correctly retrieved from the upper boundary [Snieder et al., 2006b]. Absence of sources on the lower boundary is therefore not problematic. For example, we show a numerical cross-correlation result from a 1D model, as shown in Figure 1a), with depth profiles for the relative electric permittivity and conductivity, shown in Figures $1 \mathrm{~b}$ and $1 \mathrm{c}$. We take a single transient source at $\boldsymbol{x}_{3 ; 1}$ more than $1.5 \mathrm{~m}$ above the ground surface. The receiver at $x_{3 ; A}$ is $1.5 \mathrm{~m}$ above the ground surface and the other at $\boldsymbol{x}_{3 ; B}$ is on the surface. We then cross-correlate the recorded data at these two points. The result is compared with the exact response, shown in Figure 2a. The direct wave including the near surface effects, arrives around $5 \mathrm{~ns}$ and the reflections from the subsurface boundaries at $50 \mathrm{~cm}$ depth, event around $11 \mathrm{~ns}$, and at $75 \mathrm{~cm}$, event around $15 \mathrm{~ns}$, and their multiples can also be seen. The normalized error is shown in $\%$ in Figure 2b. As can be seen in Figure 2, the response is very accurately retrieved by cross-correlation. All artifacts, due to the fact that a single source is used, are located before the first arrival in the first $2 \mathrm{~ns}$, where the largest errors are seen. These can be seen as the non-physical event in the first $2 \mathrm{~ns}$ interval in Figure 2a. This example shows that one-sided illumination [Wapenaar, 2006] can be used also for media with loss mechanisms.

\section{Concluding Remarks}

[12] From the reciprocity theorem of the time-correlation and time-convolution types, we have derived exact Green's function representations for electromagnetic fields and waves in media with losses. When the medium properties near the boundary are smoothly varying, the exact representations simplify to representations that can be used in practical applications. The theoretical example discussed here utilizes transient electric current sources in the air. They could be either noise or transient sources, which can be unknown or controlled. When these are noise sources, they can emit simultaneously but if they are transient sources, they must emit signals in a form that allows for them to be recorded separately. Examples of such sources are background radiation in a wide frequency band and satellite signals in smaller bands. In the atmosphere there are many sources of electromagnetic energy in low frequency ranges as well as in the bands used for radio waves and wireless communication (e.g. wifi and mobile telephones). From stationary phase analysis we know that not all points on the boundary are equally important. The target depth and required survey size for the receivers determines the required horizontal extent of sources on the boundary. A general good estimate would be that the sources are on a boundary that extends the receiver array on both sides by at least the target depth range. With present day emergence of ultrawideband electromagnetics components it will become possible to carry out such experiments. That would open a whole new range of electromagnetic applications. For small band signals the first electromagnetic experiments are reported by Lerosey for time reversal [Lerosey et al., 2004] and by Henty and Stancil for super resolution focusing in the frequency domain [Henty and Stancil, 2004]. Since we have formulated representations for lossy media, they can be used for diffusive electromagnetic fields and stationary electric currents. Of course all these representations can be transferred to other lossy wave types, like poroelasticity [Biot, 1956] or coupled poroelastic and electromagnetic fields [Pride, 1994], and can be used for linear diffusion processes like telluric and eddy currents or heat and viscous flow.

[13] Acknowledgments. This work is part of the research program of the Netherlands research center for Integrated Solid Earth Science (ISES).

\section{References}

Biot, M. (1956), Theory of elastic waves in a fluit saturated porous solid: I. Low frequency range, J. Acoust. Soc. Am., 28, 168-178.

Bojarski, N. (1983), Generalized reaction principles and reciprocity theorems for the wave equations, and the relationships between time-advanced and time-retarded fields, J. Acoust. Soc. Am., 74, 281-285.

Buckingham, M., B. Berkhout, and S. Glegg (1992), Imaging the ocean with ambient noise, Nature, 356, 327-329.

Campillo, M., and A. Paul (2003), Long-range correlations in the diffuse seismic coda waves, Science, 299, 547-549. 
Claerbout, J. (1968), Synthesis of a layered medium from its acoustic transmission response, Geophysics, 33, 264-269.

Cox, H. (1973), Spatial correlation in arbitrary noise fields with application to ambient sea noise, J. Acoust. Soc. Am., 54, 1289-1301.

Daneshvar, M., C. Clay, and M. Savage (1995), Passive seismmic imaging using microearthquakes, Geophysics, 60, 1178-1186.

de Hoop, A. (1995), Handbook of Radiation and Scattering of Waves, Elsevier, New York.

Derode, A., E. Larose, M. Tanter, J. de Rosny, A. Tourin, M. Campillo, and M. Fink (2003), Recovering the Green's function from field-field correlations in an open scattering medium (L), J. Acoust. Soc. Am., 113, $2973-$ 2976, doi:10.1121/1.1570436.

Draganov, D., K. Wapenaar, and J. Thorbecke (2004), Passive seismic imaging in the presence of white noise sources, Leading Edge, 23, $889-892$.

Draganov, D., K. Wapenaar, W. Mulder, and J. Singer (2006a), Seismic interferometry on background-noise field data, in Expanded Abstracts of the Society of Exploration Geophysicists, edited by J. R. Detomo Jr., pp. 590-594, Soc. of Explor. Geophys., Tulsa, Okla.

Draganov, D., K. Wapenaar, and J. Thorbecke (2006b), Seismic interferometry: Reconstructing the Earth's reflection response, Geophysics, 71, SI61-SI70.

Epifanio, C., J. Potter, G. Deane, M. Readhead, and M. Buckingham (1999), Imaging in the ocean with ambient noise: The ORB experiments, J. Acoust. Soc. Am., 106, 3211-3225.

Henty, B., and D. Stancil (2004), Multipath-enabled super-resolution for RF and microwave communication using phase-conjugate arrays, Phys. Rev. Lett., 93, 243904, doi:10.1103/PhysRevLett.93.243904.

Larose, E., G. Montaldo, A. Derode, and M. Campillo (2006), Passive imaging of localized reflectors and interfaces in open media, Appl. Phys. Lett., 88, 104103, doi:10.1063/1.2186112.

Lerosey, G., J. de Rosny, A. Tourin, A. Derode, G. Montaldo, and M. Fink (2004), Time reversal of electromagnetic waves, Phys. Rev. Lett., 92, 193904, doi:10.1103/PhysRevLett.92.193904.

Lobkis, O., and R. Weaver (2001), On the emergence of the Green's function in the correlations of a diffuse field, J. Acoust. Soc. Am., 110, 3011-3017.

Louie, J. (2001), Faster, better: Shear-wave velocity to 100 meters depth from refraction microtremor arrays, Bull. Seismol. Soc. Am., 91, 347-364.

Malcolm, A., J. Scales, and B. van Tiggelen (2004), Modeling of wave propagation in inhomogeneous media, Phys. Rev. E, 70, 015601, doi:10.1103/PhysRevE.70.015601.

Pride, S. (1994), Governing equations for the coupled electromagnetics and acoustics of porous media, Phys. Rev. B, 50, 678-696.

Ricket, J., and J. Claerbout (1999), Acoustic daylight imaging via spectral factorization: Helioseismology and reservoir monitoring, Leading Edge, 18, 957-960.

Roux, P., W. Kuperman, and the NPAL Group (2004), Extracting coherent wave fronts from acoustic ambient nosie in the ocean, J. Acoust. Soc. Am., 116, 1995-2003.

Roux, P., K. Sabra, W. Kuperman, and A. Roux (2005), Ambient noise cross correlation in free space: Theoretical approach, J. Acoust. Soc. Am., 117, $79-84$.

Sabra, K. G., P. Gerstoft, P. Roux, W. A. Kuperman, and M. C. Fehler (2005), Surface wave tomography from microseisms in southern California, Geophys. Res. Lett., 32, L14311, doi:10.1029/2005GL023155.
Schuster, G., J. Yu, J. Sheng, and J. Rickett (2004), Interferometric/daylight seismic imaging, Geophys. J. Int., 157, 838-852.

Shapiro, N. M., and M. Campillo (2004), Emergence of broadband Rayleigh waves from correlations of the ambient seismic noise, Geophys. Res. Lett., 31, L07614, doi:10.1029/2004GL019491.

Shapiro, N., M. Campillo, L. Stehly, and M. Ritzwoller (2005), Highresolution surface-wave tomography from ambient seismic noise, Science, 307, 1615-1618.

Slob, E., D. Draganov, and K. Wapenaar (2006a), Interferometric electromagnetic Green's functions representations using propagation invariants, Geophys. J. Int., in press.

Slob, E., D. Draganov, and K. Wapenaar (2006b), GPR without a source, paper presented at 11th International Conference on GPR, Ohio State Univ., Columbus, Ohio.

Snieder, R. (2004), Extracting the Green's function from the correlation of coda waves: A derivation based on stationary phase, Phys. Rev. E, 69, 046610, doi:10.1103/PhysRevE.69.046610.

Snieder, R. (2006), Retrieving the Green's function of the diffusion equation from the response to a random forcing, Phys. Rev. E, 74, doi:10.1103/PhysRevE.74.046620.

Snieder, R., J. Sheiman, and R. Calvert (2006a), Equivalence of the virtualsource method and wave-field deconvolution in seismic interferometry, Phys. Rev. E, 73, 066620, doi:10.1103/PhysRevE.73.066620.

Snieder, R., K. Wapenaar, and K. Larner (2006b), Spurious multiples in seismic interferometry of primaries, Geophysics, 71, SI111SI124

van Tiggelen, B. (2003), Green function retrieval and time reversal in a disordered world, Phys. Rev. Lett., 91, 243904, doi:10.1103/PhysRevLett.91.243904

Wapenaar, K. (2003), Synthesis of an inhomogeneous medium from its acoustic transmission response, Geophysics, 68, 1756-1759.

Wapenaar, K. (2004), Retrieving the elastodynamic Green's function of an arbitrary inhomogeneous medium by cross correlation, Phys. Rev. Lett., 93, 254301, doi:10.1103/PhysRevLett.93.254301.

Wapenaar, K. (2006), Green's function retrieval by cross-correlation in case of one-sided illumination, Geophys. Res. Lett., 33, L19304, doi:10.1029/ 2006 GL027747.

Wapenaar, K., and J. Fokkema (2006), Green's function representations for seismic interferometry, Geophysics, 71, SI33-SI46.

Wapenaar, K., E. Slob, and R. Snieder (2006), Unified Green's function retrieval by cross-correlation, Phys. Rev. Lett., 97, 234301, doi:10.1103 PhysRevLett.97.234301.

Weaver, R., and O. Lobkis (2001), Ultrasonics without a source: Thermal fluctuation correlations at MHz frequencies, Phys. Rev. Lett., 87, 134301, doi:10.1103/PhysRevLett.87.134301.

Weaver, R., and O. Lobkis (2004), Diffuse fields in open systems and the emergence of the Green's function (L), J. Acoust. Soc. Am., 116, $2731-$ 2734, doi:10.1121/1.1810232.

E. Slob and K. Wapenaar, Department of Geotechnology, Delft University of Technology, Mijnbouwstraat 120, NL-2628 RX, Delft, Netherlands. (e.c.slob@tudelft.nl; c.p.a.wapenaar@tudelft.nl) 